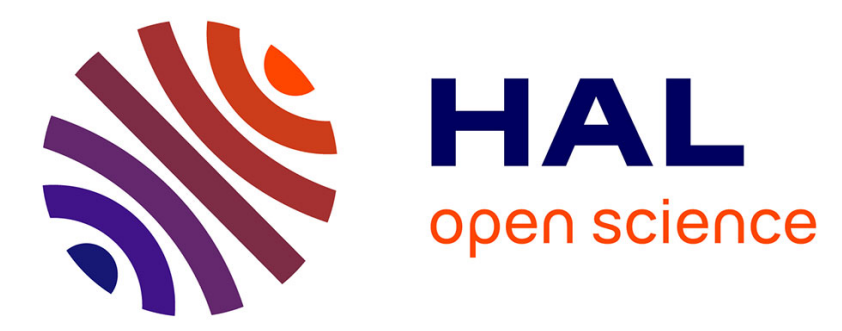

\title{
Management of T1 colorectal cancers detected at screening colonoscopy A study from the French national screening programme
}

Thomas Grainville, Jean-François Bretagne, Christine Piette, Chloé Rousseau, Martin Bordet, Mathilde Cosson, Astrid Lièvre

\section{To cite this version:}

Thomas Grainville, Jean-François Bretagne, Christine Piette, Chloé Rousseau, Martin Bordet, et al.. Management of T1 colorectal cancers detected at screening colonoscopy A study from the French national screening programme. Digestive and Liver Disease, 2020, 52 (8), pp.909-917. 10.1016/j.dld.2020.04.022 . hal-02890721

\section{HAL Id: hal-02890721 \\ https://hal.science/hal-02890721}

Submitted on 10 Jul 2020

HAL is a multi-disciplinary open access archive for the deposit and dissemination of scientific research documents, whether they are published or not. The documents may come from teaching and research institutions in France or abroad, or from public or private research centers.
L'archive ouverte pluridisciplinaire HAL, est destinée au dépôt et à la diffusion de documents scientifiques de niveau recherche, publiés ou non, émanant des établissements d'enseignement et de recherche français ou étrangers, des laboratoires publics ou privés. 
Ms. Ref. No.: DLD-20-289

MANAGEMENT OF T1 COLORECTAL CANCERS DETECTED AT SCREENING COLONOSCOPY: A STUDY FROM THE FRENCH NATIONAL SCREENING PROGRAMME

Thomas Grainville, ${ }^{a}$ Jean-François Bretagne, ${ }^{b}$ Christine Piette, ${ }^{c}$ Chloé Rousseau, ${ }^{d}$ Martin Bordet, ${ }^{a}$ Mathilde Cosson, ${ }^{c}$ Astrid Lièvre ${ }^{a, b, c, e}$

a Department of Gastroenterology, University Hospital, 35033 Rennes, France

b Rennes 1 University, 35000 Rennes, France

c ADECl 35 (Association pour le Dépistage des Cancers en Ille-et-Vilaine), 35040 Rennes, France

d Department of Biostastics, University Hospital, 35033 Rennes, France

e COSS (Chemistry Oncogenesis Stress Signaling), UMR_S 1242, Rennes, France

Corresponding author: J.-F. Bretagne; Phone: +33 607491311;

E-mail: jf.bretagne@gmail.com

\section{Funding}

None

\section{Ethics approval and consent to participate}

The colorectal cancer screening programme was declared and approved by the CNIL (Commission Nationale de l'Informatique et des Libertés) on August 30 th 2002 ( $\left.n^{\circ} 812571\right)$.

This research was approved by the CCTIRS (Comité Consultatif pour le Traitement de I'Information en Matière de Recherche dans le Domaine de la Santé).

Electronic count: 4285 words 


\section{SUMMARY}

Aim: The main aim of this study was to examine the management strategies that were used and to determine the outcomes (survival and recurrence rate) of screen-detected T1-CRC.

Methods: Medical records from 207 patients with T1-CRC diagnosed through the French national screening programme in one district from 2003 to 2015 were analysed. The 5-year overall, CRC-specific and CRC-free survival were calculated for the whole cohort and for the 3 groups treated by endoscopic resection (ER) alone, ER followed by subsequent surgery (ERSS), and primary surgery (PS).

Results: Of the 207 patients, 81 (39\%) underwent PS, and 126 (61\%) underwent primary ER, of whom $82(64 \%)$ underwent subsequent surgery. The 5-year overall and cancer-specific survival rates were $95.5 \%(95 \% \mathrm{Cl}, 90.8 ; 97.9)$ and $98.8 \%(95 \% \mathrm{Cl}, 95.4 ; 99.7 \%)$, respectively. Long-term cancer-specific mortality and recurrence crude rates were $2.4 \%$ and $5.6 \%$, respectively. The 5-year CRC-free survival rate was $96.1 \%(95 \% \mathrm{Cl}, 91.8 ; 98.1 \%)$ and did not differ among the 3 groups (ER alone, ERSS and PS).

\section{Conclusion}

This study demonstrates the good prognosis of screen-detected T1-CRC, regardless of the treatment strategy used. But, there is a room to improve the screening programme quality with regard to the management of screen-detected CRC.

Key words: Colorectal cancer screening, T1 colorectal cancer, endoscopic therapy, surgical treatment, colorectal cancer prognosis 
Abbreviations: LNM: lymph node metastasis; MDS: multidisciplinary staff; CRC: colorectal cancer;

TAE: transanal excision; CI: confidence interval; HR: hazard ratio; OR: odds ratio 


\section{Introduction}

The estimated burden of colorectal cancer (CRC) in the 28 member states of the European Union was 378,400 new cases and 173,200 deaths in 2018 , while the corresponding values for 40 countries in Europe were 499,700 and 242,500 cases, respectively [1]. There is now robust evidence that population screening can reduce cancer-specific mortality by detecting and treating disease at an early stage [2,3]. Fortunately, the implementation of mass screening for CRC is increasing in Europe, with roll-outs ongoing or completed in 17 member states in 2016 compared to only five in 2007 [4]. A national CRC screening programme was implemented in France at the beginning of 2003 and was then generalized to the whole country in 2008; this program includes the biennial faecal blood test Hemoccult II, which was replaced by the OC-Sensor immunochemical test in 2015 [5]. In one district of Brittany, we demonstrated the benefit of the switch from a guaiac method to immunochemical testing in terms of increased participation and detection of CRC and advanced neoplasia [6]. If the participation rate, the positivity rate, the detection rate of colorectal lesions and the positive predictive value for adenomas and CRC are well-studied key performance indicators of CRC screening programmes [7], the management and the prognosis of screen-detected lesions are also quality markers of screening programmes [8,9]; however, these aspects have been studied very little. Based on a population study, we were among the first to report great variations in the management of nonmalignant screen-detected polyps, particularly for those $\geq 20 \mathrm{~mm}$ in size; patients were referred for surgical resection in $20 \%$ of cases, with adjusted rates ranging from 0 to $46 \%$, depending on the gastroenterologist performing the screening colonoscopy [10]. However, there are few reported data on the management of 
cancers diagnosed through CRC screening programmes, which are mainly early cancers being the basis for the reduction of CRC-specific mortality in screening programmes [11-13].

Therefore, the primary aim of this study was to use a population-based approach to examine the management strategies and the outcomes (survival and recurrence rate) of all patients with pT1 screen-detected CRC in one district of Brittany over a 13-year period. The secondary objectives were i) to identify factors associated with the choice of an initial endoscopic or surgical treatment, ii) to assess whether cases had been the subject of a multidisciplinary staff (MDS) presentation and examine, in case of primary endoscopic resection, if the decision of a complementary surgery or not was taken according to the recommendations and iii) to determine the risk factors associated with disease recurrence.

\section{Population \& methods}

\subsection{Study population}

This study was a retrospective analysis of a prospective cohort that included all patients with pT1 adenocarcinoma diagnosed by colonoscopy following a positive faecal test through the national screening programme in the 'Ille-et-Vilaine' district from 2003 to 2015. Details on the screening programme were previously reported [6]. pT1 carcinomas have invaded the muscularis mucosae into the submucosa but not into the muscularis propria; the endoscopic or surgical treatment of these carcinomas is controversial [10]. In this 13-year period, 565288 faecal blood tests were performed. There were 14215 (2.5\%) positive tests that were followed by 13245 colonoscopies (93.2\%), and 1060 cases of cancer were diagnosed, which included 207 (19.5\%) cases of pT1 adenocarcinomas. Among pT1 carcinomas, 181 (87.4\%) were detected by guaiac-based faecal testing, while $26(12.6 \%)$ were detected by 
FIT. The FIT selected by the French Health Authorities was the OC-Sensor ${ }^{\mathrm{TM}}$ test (Eiken, Tokyo, Japan) which has been used from May 2015 and was considered to be positive at the cut-off of $30 \mu \mathrm{g}$ of haemoglobin per gram of faeces.

Demographic and baseline clinical features, colonoscopy results, pathological features of endoscopic biopsy specimens and from endoscopic or surgical resection specimens, therapeutic management and follow-up data were collected. All data related to the screening programme were prospectively collected in the database of the district ( $A D E C I 35)$, while information on patient follow-up, when missing, was requested from general practitioners and gastroenterologists. Whether cases had been the subject of a MDS presentation was examined in patient medical records.

\subsection{Colonoscopy data}

The following data were collected: the date of colonoscopy, the endoscopy centre where the colonoscopy was performed, the gastroenterologist who performed the colonoscopy and potential complications that occurred during and within 30 days after the colonoscopy by using the severity grading system reported by the American Society for Gastrointestinal Endoscopy [14]. The following characteristics of malignant polyps were collected: polyp morphology (pedunculated or sessile), size when precisely measured or classified into 3 categories (<20 mm, $20-29 \mathrm{~mm}$ or $\geq 30 \mathrm{~mm}$ ), polyp location (proximal, distal, or rectal) and modalities for removal (resection technique, en bloc or piecemeal resection), as well as the number of associated polyps. Polyposis was defined as the presence of at least 10 polyps. The location of the cancer was defined as proximal or distal if it was located proximally or distally to the splenic flexure, respectively. 
When the endoscopic resection of carcinoma was not performed during the initial diagnostic colonoscopy, we investigated whether the patient was referred to another endoscopist for therapeutic colonoscopy.

\subsection{Pathology features}

For this analysis, pT1 cancers were categorized retrospectively as having a low-risk or highrisk of lymph node metastases (LNM) according to the absence or presence of at least one of the following prognostic pathological criteria notified in the pathological report: poor differentiation, venous and lymphatic invasion, tumour budding, submucosal invasion depth $\geq 1,000 \mu \mathrm{m}$ for sessile polyps or Haggitt level of invasion $\geq 3$ for pedunculated polyps, and margin involvement with a clearance of $1 \mathrm{~mm}$ or less [15]. We also recorded the width ( $\leq$ or $>4,000 \mu \mathrm{m})$ of submucosal invasion.

\subsection{Surgical characteristics}

We retrospectively retrieved the following data from the medical records of patients who underwent surgery: date and reason for surgery, type of operation and complications within 30 days of surgery according to the Clavien-Dindo classification [16]. In addition to the pathological features that were already described for malignant polyps resected by endoscopy, we collected data on LNM and residual tumour on surgical specimens for those who underwent subsequent surgery after endoscopic resection or an initial operation. 


\subsection{Outcomes and statistical analysis}

The close follow-up of patients aimed to assess mortality related or not related to tumour progression and local or metastatic recurrence. The primary outcomes included determining the 5-year overall, cancer-specific and recurrence-free survival as well as the long-term CRCspecific mortality and recurrence crude rates for the whole cohort and the subgroups defined by the management strategy. The secondary outcomes were to identify the factors associated with particular management strategies (primary endoscopic or surgical resection) and the risk factors associated with disease recurrence. In patients who had a primary endoscopic resection, the decision to perform a complementary surgery was considered in accordance with recommendations $[17,18]$ if the pT1 cancer was at high-risk of LNM as defined above.

Means and standard deviations (SDs) were calculated for continuous variables, and frequencies and percentages were calculated for categorical variables. Groups were compared by the $t$ test or the Wilcoxon rank-sum test for continuous variables and by the $\chi^{2}$ test or the Fisher exact test for categorical variables.

Recurrence-free survival curves were generated by using the Kaplan-Meier product limit method, and the log-rank test was used to compare survival between the endoscopic therapy group and the surgery groups.

Univariate and multivariate Cox proportional hazards regression models were used to assess the factors associated with cancer recurrence and the treatment modalities. Factors associated with the therapeutic strategy were investigated using univariate logistic regressions. Potential covariates $(p \leq 0.20)$ were placed into a multivariable logistic regression 
model that was performed by using a stepwise backward elimination. To investigate the influence of the endoscopist, we compared the results from endoscopists who performed more than 10 colonoscopies that resulted in a pT1 cancer diagnosis to the results of other endoscopists in the univariate and multivariate analysis. Odd ratios (ORs) and the corresponding $95 \% \mathrm{Cls}$ were used to express the association between the studied features and outcomes. For univariate analysis, the association of each variable with cancer recurrence was tested by a Cox model. To identify the clinical, colonoscopic and pathological features associated with disease recurrence, significant variables with $p \leq 0.20$ were included in the multivariable analysis that was performed by using a stepwise backward elimination. Hazard ratios (HRs) and the corresponding 95\% confidence intervals (Cls) were used to express the association between the studied features and outcomes.

All analyses were performed using the statistical software program SAS version 9.4 (SAS Institute Inc., Cary, North Carolina, USA). Statistical significance was defined as $p \leq 0.05$.

\section{Results}

\subsection{Characteristics of the cohort and the management strategy}

The characteristics of 207 patients, malignant polyp and colonoscopy data are given in Table 1. Among the 207 patients, 126 underwent primary endoscopic resection, while 81 underwent primary surgery. Pathological features are given in Table $\mathbf{2}$ for both groups. In 82 of the 126 endoscopically treated patients, endoscopic resection was followed by subsequent surgery; thus, $44(21.3 \%)$ patients were treated by endoscopic resection alone and $163(78.7 \%)$ were treated by surgery alone or surgery preceded by endoscopic therapy. 
Four patients who underwent complementary surgery had already undergone surgery. Of the 81 patients who underwent primary surgery, 74 had pathological evidence of carcinoma in the endoscopic biopsy specimens.

Among the 126 patients who underwent primary endoscopic resection, polyp-cancer resection was performed during the diagnostic colonoscopy in all patients, except for 8 (6.3\%) patients who were either referred to a skilled endoscopist $(n=5)$ or treated by the same endoscopist during an additional colonoscopy $(n=3)$. Endoscopic resection was followed by a complication in $13(10.3 \%)$ patients, with mild, moderate and severe grades in 8,3 and 2 patients, respectively (Table 3 ). Patient management was discussed by a MDS in 64 of the 126 (51\%) patients. In all patients except for one, the staff's decision was implemented.

Among the 81 patients who underwent primary surgery, $72(88.9 \%)$ patients underwent surgery because endoscopic resection was considered unsuitable by the endoscopist, 7 (8.6\%) patients underwent surgery after an attempt of the endoscopic resection, and 2 (2.5\%) underwent surgery because of synchronous polyposis. The operations that were performed and the post-operative complications are given in Table 3. A mean of $13.1 \pm 9.4$ lymph nodes were resected, but fewer than 12 lymph nodes were harvested in 31 (38.2\%) patients, and 7 patients did not undergo lymph node dissection. Among the 74 patients who underwent lymph node dissection, LNM was present in 7 (9.5\%) patients. The patient management of 38 (47\%) patients was discussed by an MDS. 
Finally, 86 patients underwent subsequent surgery: 81 (94.2\%) after primary endoscopic resection and $4(4.7 \%)$ after primary surgery. The operations performed and post-operative complications are given in Table 3. Residual adenoma or adenocarcinoma were observed on surgical specimens in 5 of the 82 patients with endoscopic resection and in 2 of the 4 patients who had already undergone surgery. A mean of $13.2 \pm 8.9$ lymph nodes were resected, but fewer than 12 lymph nodes were harvested in 31 (38.7\%) patients and 4 patients did not undergo lymph node dissection, which included 2 patients who had already undergone surgery. Among the 82 patients who underwent lymph node dissection, 9 (11\%) had LNM.

\subsection{Outcomes}

\subsubsection{Main outcomes}

Follow-up data were available for 198 patients, and the median follow-up was 6.7 \pm 3.9 years. Thirty-six (18.2\%) patients died during the 13 -year period of follow-up, and 5 (2.4\%) of these deaths were related to cancer recurrence. The 5-year overall and cancerspecific survival rates were $95.5 \%(95 \% \mathrm{Cl}, 90.8 ; 97.9)$ and $98.8 \%(95 \% \mathrm{Cl}, 95.4 ; 99.7 \%)$, respectively. Tumour recurrence occurred in 11 patients (5.6\%) (8, 2 and 1 patients in the groups treated by primary surgery, endoscopic resection only and primary endoscopic resection followed by subsequent surgery, respectively), which included 4 local and 7 metastatic recurrences (Table 4). Among the 8 patients who recurred after primary surgery, 3 patients underwent TAE and did not undergo subsequent surgery despite being at high-risk for LNM. The two patients who had disease recurrence after receiving only endoscopic therapy did not undergo subsequent surgery despite having unsafe or invaluable margins on 
the resection specimen. The only patient who had recurrence following subsequent surgical resection after endoscopic therapy had LNM based on the surgical resection specimen. The time to recurrence was less than 2.5 years in 6 patients. Among the 11 patients who relapsed, 6 died, including 5 cancer-related deaths and one death due to heart failure. The 5year recurrence-free survival rate was $96.1 \%$ (95\% Cl, 91.8; 98.1\%) (Fig. 1A), and the 5-year recurrence-free survival rate was not significantly different between patients treated by primary endoscopic resection $(96.9 \%$ [95\% Cl, 90.4; 99\%]) and treated with primary surgery (94.9\% [95\% Cl, 87; 98.1\%]) ( $p=0.059)$ (Fig. 1B). The 5-year recurrence-free survival also was not significantly different among the 3 subgroups defined by the treatment strategy: endoscopic resection alone $(93.8 \%[95 \% \mathrm{Cl}, 76.7 ; 98.5 \%]$, endoscopic resection with subsequent surgery $(98.5 \%[95 \% \mathrm{Cl}, 90 ; 99.8 \%]$ and primary surgery $(94.9 \%[95 \% \mathrm{Cl}, 87$; 98.1\%] (log rank, $p=0.12$ ) (Fig. 1C). The distribution of recurrences and cancer-related deaths according to cancer site are given in Table 4.

\subsubsection{Secondary outcomes}

\subsubsection{Factors associated with either primary endoscopic or surgical treatment}

In multivariable analysis, the three factors independently associated with surgical resection were polyp size $>20 \mathrm{~mm}$, sessile morphology of the polyp-cancer and a date of diagnosis before 2007 (Table 5).

\subsubsection{Factors associated with subsequent surgery following primary endoscopic} resection

Among the different features considered when deciding whether to perform subsequent surgery, involved, unsafe or invaluable resection margins were the only risk 
factor significantly $(p<0.0001)$ associated with surgery in univariate and multivariate analysis.

Among the 44 patients who underwent only endoscopic resection, 7 (15.9\%) patients did not undergo subsequent surgery, although they were at high risk for LNM. Among the 82 patients who underwent complementary surgery after endoscopic resection, 15 (18.3\%) were at low risk for LNM.

\subsubsection{Factors associated with disease recurrence}

In univariate analysis, the Cox model showed that piecemeal endoscopic resection, involved or unsafe resection margins and sessile morphology of the polyp cancer were significantly associated with disease recurrence. In multivariate analysis, involved or unsafe resection margins $(\mathrm{OR}=15.3[95 \% \mathrm{Cl}, 2.8 ; 83.7], \mathrm{p}=0.0018)$ and primary surgery $(\mathrm{OR}=12.2$ $[95 \% \mathrm{Cl}, 2.4 ; 63.6], \mathrm{p}=0.0029)$ were the only factors that were independently associated with recurrence. When the 6 patients who underwent primary transanal excision (TAE) were considered to have undergone endoscopic resection (as it does not comprise any lymph node dissection), primary surgery was no longer found to be a significant risk factor for disease recurrence (data not shown).

\section{Discussion}

The primary aim of the present population-based study was to examine the management of pT1 screen-detected colorectal carcinoma and to assess the long-term outcomes of 207 cases of pT1 screen-detected colorectal carcinoma over a 13-year period. The present study emphasizes the good prognosis of pT1 screen-detected colorectal carcinoma patients. Thus, the 5-year overall, cancer-specific and recurrence-free survival of the cohort was $95.5 \%$, 
$98.8 \%$ and $96 \%$, respectively, while the long-term cancer-specific mortality and recurrence crude rates were $2.4 \%$ and $5.6 \%$, respectively; these findings were in perfect agreement with those from a Scottish study (2\% and $6 \%$, respectively) [13]. The excellent prognosis of screen-detected early carcinoma is an exciting outcome, highlighting the beneficial effect of screening programmes in reducing cancer-specific mortality [2,3]. Additionally, we demonstrated that 5-year recurrence-free survival did not differ significantly among the three subgroups defined by the treatment strategy: endoscopic therapy alone, endoscopic therapy with subsequent surgery or primary surgery $(93.8 \%, 98.5 \%$ and $94.9 \%$, respectively). These results confirmed those of a North-Western Italian study that also examined a screening programme [12]. In contrast, in a large American population-based study conducted outside of the screening setting, a better recurrence-free survival was reported in the group of surgical patients, a result that could be explained by the fact that among the surgical patients with pT1 carcinoma, those with LMN were not included [19]. Although there was no significant difference in 5-year recurrence-free survival of surgical and nonsurgical patients found in our study, logistic regression analysis initially found that primary surgery was an independent risk factor for disease recurrence, in addition to an involved, unsafe or invaluable resection margin. However, it was not confirmed by a new analysis in which the 6 patients who underwent TAE were moved to the group of patients who have undergone endoscopic resection.

Interestingly, the 5 patients who had disease recurrence after receiving only endoscopic therapy (including TAE) did not undergo subsequent surgery despite having unsafe or invaluable margins on the resection specimen $(n=3)$ or other risk factor of LNM (vascular emboli and submucosal invasion depth $\geq 1,000 \mu \mathrm{m})(\mathrm{n}=2)$. Moreover, 4 of the 6 patients who had disease recurrence after surgery (including one subsequent surgery after primary 
endoscopic resection) had LNM or fewer than 12 lymph nodes harvested on the surgical resection specimens. These data highlight once more the prognostic value of pathological features, especially a positive resection margin [20], which was an independent risk factor for disease recurrence in our study, but also a poor differentiation, vascular invasion, tumour budding, a submucosal invasion depth $\geq 1,000 \mu \mathrm{m}$ for sessile polyps or Haggitt level of invasion $\geq 3$ for pedunculated polyps as well as lymph node involvement for patients undergoing surgery. These histological parameters were not found as prognostic factors in the present study, which may be explained by the large proportion of missing data, particularly concerning tumour budding (90\%), Haggitt classification (66\%) and submucosal invasion depth (58\%). Concerning tumour budding, however, no authoritative endorsements recommended its inclusion in the pathology report at the time of the present study because of the heterogeneity of definitions and measurement methods as well as its poor reproducibility and controversial prognostic impact [15]. A recent meta-analysis has since demonstrated its role as a risk factor for nodal metastasis specifically in pT1 colorectal cancer [21]. A revision of histological specimens by a gastrointestinal pathologist, performing a re-evaluation of all risk factors for lymph node metastasis, would have been interesting in our study as it has been shown to significantly modify risk assessment of pT1 endoscopically resected colorectal cancer and their clinical management [22].

In our study, it should be added that lymph node involvement is most likely underestimated in patients who have had a surgery because $38 \%$ of patients had less than 12 lymph nodes removed in the primary and subsequent surgery groups and $9 \%$ and $5 \%$ of them respectively, did not have any lymph node dissection. However, lymph node invasion is one of the factors, if not the most important prognostic factor in resected colorectal cancers [23]. Taking into account these findings, which reflect real-life practices, we can easily 
consider that the proportion of high-risk pT1 cancers was underestimated in our study and that the incidence of disease recurrence would have been much lower if the recommendations have been implemented. In view of these limitations, it is difficult to draw any robust conclusion about the best therapeutic strategy to offer in the case of pT1 cancer.

Compared to similar studies, the direct surgical referral rate $(39 \%)$ in this study was slightly higher than that in Italian screening programmes (24-30\%) [11,12] but was slightly lower than that in the UK (43\%) [24], with a slightly lower referral rate for radical surgery (57-69\% and $53 \%$, respectively, compared to $64 \%)$. Nevertheless, the overall surgical rate $(78.7 \%)$ that we found was similar to that of an Italian study [12]. To investigate which variables were most relevant to surgical decision making, the multivariate analysis highlighted the role of polyp-cancer morphology and the date of colonoscopy. Thus, $75 \%$ of patients who underwent primary surgery had sessile polyps, $49.4 \%$ of malignant polyps were $\geq 30 \mathrm{~mm}$, while the patients screened after 2007 were almost 3 times more likely to undergo primary endoscopy than surgical resection. This result could be related to the introduction of new and better trained gastroenterologists and/or to the improvement of gastroenterologists through post-graduate training. However, the gastroenterologist who performed the screening colonoscopy was not independently associated with the choice of treatment based on multivariate analysis, although some gastroenterologists had significantly lower odds ratios for endoscopic resection rates than the gastroenterologist with the highest endoscopic resection rate based on univariate logistic regression analysis.

Given that only $30 \%$ of patients who underwent primary surgery had high-risk pathological features of LNM or had LNM on surgical specimens, one may wonder why the other patients 
were directly referred to a surgeon without a referral to expert endoscopists. There was no clear, determinable reason why endoscopic resection was not considered suitable based on the diagnostic colonoscopy. One could speculate that the proportion of primary surgery would have decreased if patients had been referred to endoscopists with more expertise for the characterization and treatment of complex polyps. Indeed, it has been shown in the UK that the rate of primary surgical resection was lower for T1-CRC identified from screening than for those resulting from diagnosis [25]. In the UK but not in France, endoscopists are accredited for screening colonoscopy, and polypectomy skills are a part of the national accreditation process for colonoscopy certification [26]. The relatively high rate of postendoscopic complications observed in the present study, compared to the rates reported in the English National Health Service Bowel Cancer Screening Programme [27,28], could be regarded as a marker of less experience in polypectomy. Similarly, in their meta-analysis of 21 population-based studies, Reumkens et al. reported low rates of post-polypectomy perforation and haemorrhage, equal to 0.8 and 9.8 p.1000 colonoscopies including all types of polyps [29]. However, in their meta-analysis assessing the efficacy and safety of endoscopic resection for large colorectal polyps ( $\geq 20 \mathrm{~mm}$ ), $58 \%$ of which being invasive cancers, Hassan et al. reported much higher values for perforation and bleeding $(1.5 \%, 6.5 \%$, respectively) [30]. In their study specifically devoted to endoscopic resection of colorectal cancers T1, Overwater et al. reported post-endoscopic complication rates of $6.5 \%$, including 4.1\% for bleeding, in a series of 339 high-risk T1 cancer cases requiring secondary surgery [31]. In another recent multicentric study from Netherlands also devoted to T1 carcinomas, the rate of post-endoscopic adverse events was 5.5\%, including 3.7\% for bleeding [32]. Even if the authors stated that the complication rate was similar to that observed for adenomas, we argue that the safety of endoscopic resection remains to be proven for early, but invasive 
CRCs. One could also speculate from our findings that systematically discussing the medical records of polyp patients with a MDS could also help to reduce surgical referrals. The choice between endoscopic or surgical strategies is not insignificant since surgery leads to an increase in costs for screening programme managers and in complications for tested patients [33]. Furthermore, from the patient's perspective, endoscopic treatment provides a quicker recovery than surgery and does not provoke more fear of cancer recurrence [34]. Another argument supporting primary endoscopic resection is that even in cases of high-risk T1-CRC, there was not an increased risk of disease recurrence or LNM reported after secondary surgery compared with primary surgery [31,35]. The only risk factor associated with subsequent surgery following endoscopic therapy identified in the present study was involved, unsafe or invaluable resection margins, which was in agreement with other studies [11]. However, it is noteworthy that $18.3 \%$ of the 82 patients did not have any pathological risk factors. Among them, none had been the subject of a MDS presentation.

The main limitations of the present study were its retrospective nature and the suboptimal collection of data regarding some histopathology items as previously detailed. These limitations could explain why some pathological features, such as budding, were not found to be significantly associated with disease recurrence. However, progress has been noted over time; for example, the depth of submucosal invasion was not recorded before 2007 in $92.3 \%$ of cases but was recorded in $89.7 \%$ of cases after 2007 . The use of standardized forms that are now recommended for pathology reports of endoscopically resected pTis and pT1 colorectal polyps by French guidelines [36] should improve the quality of pathology reports in the future. The reassessment of pathology that we were unable to perform for this study could have modified the risk assessment, as already mentioned [22]. Because deviant cases 
had not been the subject of a MDS presentation, we cannot tell from the records review whether the absence of additional surgery for some high-risk patients or the occurrence of surgery for some low-risk patients was a patient's decision or that of the gastroenterologist. It is also possible that the existence of co-morbidity factors, not identified in our study, may have influenced the decision not to operate or to reoperate on some high-risk patients. Finally, we cannot extrapolate our findings to the whole country, even if we know that our previous findings regarding nonmalignant polyps were replicated in other districts in France [37]. This study also had several strengths, particularly the completeness of the data collected concerning colonoscopy and surgical findings and the median length of 6.5 years for follow-up, never reported so far, which may explain the observed rate of disease recurrence. Of the 11 recurrences, 5 were diagnosed after 54 months.

In conclusion, the present study shows that the prognosis of early (pT1) screen-detected CRC is excellent, regardless of the management strategy used. This study demonstrates that a large proportion of patients with pT1-CRC underwent direct or subsequent surgery, while surgery could probably have been avoided to a certain extent by referring the patient to a skilled endoscopist. The proportion of patients who underwent primary surgery decreased with time, suggesting an improvement in the training of gastroenterologists in endoscopic resection techniques. Our study also shows that there is room for improvement in the quality of pathology reports and for systematically discussing patient records with a MDS to define the best treatment strategy for each patient. 


\section{Acknowledgements}

The authors acknowledge the contributions of the general practitioners, gastroenterologists and surgeons in the district of Ille-et-Vilaine. They also acknowledge the staff in charge of the database at the screening centre, particularly Samuel Foucrit, a computer scientist. 


\section{REFERENCES}

1. Ferlay J, Colombet M, Soerjomataram I, et al. Cancer incidence and mortality patterns in Europe: Estimates for 40 countries and 25 major cancers in 2018. Eur J Cancer 2018;103:356-87.

2. Zorzi M, Fedeli U, Schievano E, et al. Impact on colorectal cancer mortality of screening programmes based on the faecal immunochemical test. Gut 2015;64:78490.

3. Levin TR, Corley DA, Jensen CD, et al. Effects of Organized Colorectal Cancer Screening on Cancer Incidence and Mortality in a Large Community-Based Population. Gastroenterology 2018;155:1383-91.

4. Basu P, Ponti A, Anttila A, et al. Status of implementation and organization of cancer screening in The European Union Member States-Summary results from the second European screening report. Int J Cancer 2018;142:44-56.

5. Inca. Le Programme de dépistage organisé du cancer colorectal en France. https://www.e-cancer.fr/professionnels-de-sante/depistage-et-detectionprecoce/depistage-du-cancer-colorectal/le-programme-de-depistage-organise. 
6. Bretagne JF, Piette $C$, Cosson $M$, et al. Switching from guaiac to immunochemical faecal occult blood test increases participation and diagnostic yield of colorectal cancer screening. Dig Liver Dis 2019;51:1461-9.

7. Senore C, Basu P, Anttila A, et al. Performance of colorectal cancer screening in the European Union Member States: data from the second European screening report. Gut 2019;68:1232-44.

8. Segnan N, Patnick J, von Karsa L. European guidelines for quality assurance in colorectal cancer screening and diagnosis. Luxembourg: Publications Office of the European Union, 2010. http://www.kolorektum.cz/res/file/guidelines/CRCscreening-guidelines-EC-2011-02-03.pdf

9. Steele RJ, Pox C, Kuipers EJ, et al. European guidelines for quality assurance in colorectal cancer screening and diagnosis. First Edition--Management of lesions detected in colorectal cancer screening. Endoscopy 2012;44 Suppl 3:SE140-50.

10. Le Roy F, Manfredi S, Hamonic S, et al. Frequency of and risk factors for the surgical resection of nonmalignant colorectal polyps: a population-based study. Endoscopy 2016;48:263-70.

11. Fasoli R, Nienstedt R, De Carli N, et al. The management of malignant polyps in colorectal cancer screening programmes: a retrospective Italian multi-centre study. Dig Liver Dis 2015;47:715-9. 
12. Senore C, Giovo I, Ribaldone DG, et al. Management of Pt1 tumours removed by endoscopy during colorectal cancer screening: Outcome and treatment quality indicators. Eur J Surg Oncol 2018;44:1873-9.

13. Richards $\mathrm{CH}$, Ventham NT, Mansouri D, et al. An evidence-based treatment algorithm for colorectal polyp cancers: results from the Scottish Screen-detected Polyp Cancer Study (SSPoCS). Gut 2018;67:299-306.

14. Cotton PB, Eisen GM, Aabakken L, et al. A lexicon for endoscopic adverse events: report of an ASGE workshop. Gastrointest Endosc 2010;71:446-54.

15. Quirke P, Risio M, Lambert R, et al. Quality assurance in pathology in colorectal cancer screening and diagnosis-European recommendations. Virchows Arch 2011;458:1-19.

16. Clavien PA, Barkun J, de Oliveira ML, et al. The Clavien-Dindo classification of surgical complications: five-year experience. Ann Surg 2009;250:187-96.

17. Williams JG, Pullan RD, Hill J, et al. Management of the malignant colorectal polyp: ACPGBI position statement. Colorectal Dis 2013;15 Suppl 2:1-38.

18. Lecomte T, André T, Bibeau F, et al. [Non-metastatic colonic cancer]. Thésaurus National de Cancérologie Digestive, 2019. https://www.snfge.org/tncd et http://www.tncd.org 
19. Mounzer R, Das A, Yen RD, et al. Endoscopic and surgical treatment of malignant colorectal polyps: a population-based comparative study. Gastrointest Endosc 2015;81:733-40.

20. Belderbos TD, van Erning FN, de Hingh IH, et al. Long-term Recurrence-free Survival After Standard Endoscopic Resection Versus Surgical Resection of Submucosal Invasive Colorectal Cancer: A Population-based Study. Clin Gastroenterol Hepatol 2017;15:403-11.

21. Cappellesso R, Luchini C, Veronese N, et al. Tumor budding as a risk factor for nodal metastasis in pT1 colorectal cancers: a meta-analysis. Hum Pathol 2017;65:62-70.

22. Rampioni Vinciguerra GL, Antonelli G, Citron F, et al. Pathologist second opinion significantly alters clinical management of pT1 endoscopically resected colorectal cancer. Virchows Arch 2019;475:665-8.

23. André T, Boni C, Mounedji-Boudiaf L, et al. Multicenter International Study of Oxaliplatin/5-Fluorouracil/Leucovorin in the Adjuvant Treatment of Colon Cancer (MOSAIC) Investigators. Oxaliplatin, fluorouracil, and leucovorin as adjuvant treatment for colon cancer. N Engl J Med 2004;350:2343-51.

24. Gill MD, Rutter MD, Holtham SJ. Management and short-term outcome of malignant colorectal polyps in the north of England. Colorectal Dis 2013;15:169-76. 
25. Dattani M, Crane S, Battersby NJ, et al. Variations in the management of significant polyps and early colorectal cancer: results from a multicentre observational study of 383 patients. Colorectal Dis 2018;20:1088-96.

26. Subramaniam S, Bhandari P. Competency in polypectomy: when desirable becomes essential. Gastrointest Endosc 2018;87:645-7.

27. Derbyshire E, Hungin P, Nickerson C, et al. Post-polypectomy bleeding in the English National Health Service Bowel Cancer Screening Programme. Endoscopy 2017;49:899-908.

28. Derbyshire E, Hungin P, Nickerson C, et al. Colonoscopic perforations in the English National Health Service Bowel Cancer Screening Programme. Endoscopy 2018;50:861-70.

29. Reumkens A, Rondagh EJA, Baker M, et al. Post-colonoscopy complications: a systematic review, time-trends, and meta-analysis of population-based studies. Am J Gastroenterol 2016;111:1092-101.

30. Hassan C, Repici A, Sharma P, et al. Efficacy and safety of endoscopic resection of large colorectal polyps: a systematic review and meta-analysis. Gut 2016;65:806-20.

31. Overwater A, Kessels K, Elias SG, et al. Endoscopic resection of high-risk T1 colorectal carcinoma prior to surgical resection has no adverse effect on long-term outcomes. Gut 2018;67:284-90. 
32. van de Ven SEM, Backes $Y$, Hilbink $M$, et al. Periprocedural adverse events after endoscopic resection of T1 colorectal carcinomas. Gastrointest Endosc 2020,91:14252 e3.

33. Law R, Das A, Gregory D, et al. Endoscopic resection is cost-effective compared with laparoscopic resection in the management of complex colon polyps: an economic analysis. Gastrointest Endosc 2016;83:1248-57.

34. Dang H, de Vos Tot Nederveen Cappel WH, van der Zwaan SMS, et al. Quality of life and fear of cancer recurrence in T1 colorectal cancer patients treated with endoscopic or surgical tumor resection. Gastrointest Endosc 2019;89:533-44.

35. Rickert A, Aliyev R, Belle S, et al. Oncologic colorectal resection after endoscopic treatment of malignant polyps: does endoscopy have an adverse effect on oncologic and surgical outcomes? Gastrointest Endosc 2014;79:951-60.

36. Selves J, Terris B, Cazals-Hatem D, et al. [Guidelines for the management of malignant colo-rectal polyps (pTis and pT1) treated by endoscopic resection]. Ann Pathol 2018;38:331-7.

37. Denis B, Gendre I, Lacroute J, et al. Management of nonmalignant colorectal polyps of $20 \mathrm{~mm}$ or more in size through the colorectal cancer screening organized programme with the immunochemical test. Journées Francophones 
d'Hépatogastroentérologie et d'Oncologie Digestive, CO.06 - Paris 2018.

https://www.snfge.org/content/traitement-des-polypes-colorectaux-benins-de-20-

$\underline{\text { mm-et-plus-du-programme-de-depistage }}$ 


\section{Figure legends}

Fig. 1A

Long-term recurrence-free survival of the whole cohort

Fig. 1B

Long-term recurrence-free survival in patients primarily treated by endoscopic resection or surgical resection

Fig. 1C

Long-term recurrence-free survival in patients treated by endoscopic resection alone, endoscopic resection followed by subsequent surgery, or primary surgery 